\title{
Influence of weak layer heterogeneity and slab properties on slab tensile failure propensity and avalanche release area
}

\author{
J. Gaume ${ }^{1}$, G. Chambon ${ }^{2,3}$, N. Eckert ${ }^{2,3}$, M. Naaim ${ }^{2,3}$, and J. Schweizer ${ }^{1}$ \\ ${ }^{1}$ WSL Institute for Snow and Avalanche Research SLF, Davos, Switzerland \\ ${ }^{2}$ Irstea, UR ETGR, Grenoble, France \\ ${ }^{3}$ Université Grenoble Alpes, Grenoble, France
}

Correspondence to: J. Gaume (gaume@slf.ch)

Received: 29 October 2014 - Published in The Cryosphere Discuss.: 5 December 2014

Revised: 12 March 2015 - Accepted: 19 March 2015 - Published: 27 April 2015

\begin{abstract}
Dry-snow slab avalanches are generally caused by a sequence of fracture processes, including failure initiation in a weak snow layer underlying a cohesive slab followed by crack propagation within the weak layer (WL) and tensile fracture through the slab. During past decades, theoretical and experimental work has gradually increased our knowledge of the fracture process in snow. However, our limited understanding of crack propagation and fracture arrest propensity prevents the evaluation of avalanche release sizes and thus impedes hazard assessment. To address this issue, slab tensile failure propensity is examined using a mechanically based statistical model of the slab-WL system based on the finite element method. This model accounts for WL heterogeneity, stress redistribution by slab elasticity and possible tensile failure of the slab. Two types of avalanche release are distinguished in the simulations: (1) full-slope release if the heterogeneity is not sufficient to stop crack propagation and trigger a tensile failure within the slab; (2) partial-slope release if fracture arrest and slab tensile failure occur due to the WL heterogeneity. The probability of these two release types is presented as a function of the characteristics of WL heterogeneity and the slab. One of the main outcomes is that, for realistic values of the parameters, the tensile failure propensity is mainly influenced by slab properties. Hard and thick snow slabs are more prone to wide-scale crack propagation and thus lead to larger avalanches (full-slope release). In this case, the avalanche size is mainly influenced by topographical and morphological features such as rocks, trees, slope curvature and the spatial variability of the snow depth as often claimed in the literature.
\end{abstract}

\section{Introduction}

Dry-snow slab avalanches are generally caused by a sequence of fracture processes including (1) failure initiation in a weak snow layer underlying a cohesive slab, (2) crack propagation within the weak layer (WL) and (3) tensile fracture through the slab which leads to its detachment (McClung, 1979; Schweizer et al., 2003). During the past decades, theoretical and experimental studies have gradually enhanced the knowledge of the fracture process in snow, allowing a better estimation of snowpack stability (McClung, 1979; Jamieson and Johnston, 1990; Föhn et al., 1998; Jamieson and Johnston, 1998, 2001; Schweizer et al., 2006; Sigrist and Schweizer, 2007). Despite these advances, the limited understanding of crack propagation and fracture arrest propensity still limits the evaluation of the avalanche size and thus impedes avalanche forecasting and hazard mapping. This limitation is, inter alia, due to the multi-scale spatial variability of the snowpack and the complex microstructure of snow.

Avalanche hazard mapping procedures have recently seen growing popularity of coupled statistical-deterministic models in order to evaluate the runout distance distribution and the probability of exceedance of a threshold pressure at any location of the runout zone (Barbolini et al., 2000; Naaim et al., 2003; Ancey et al., 2004; Eckert et al., 2007, 2008, 2010). These coupled models require the evaluation of the release volume: a combination of the release depth and area. For the evaluation of the release depth, empirical techniques already exist (Swiss guidelines, Salm et al., 1990), and more recently a coupled statistical-mechanical model was proposed by Gaume et al. (2012) and Gaume et al. (2013) 
that takes into account both mechanical and meteorological factors in a probabilistic framework. However, the precise position of the release area and the evaluation of its spatial extent have been less investigated. Maggioni et al. (2002) and Maggioni and Gruber (2003) analyzed a database of well-documented avalanche events with respect to several topographic characteristics and showed that the mean slope angle, the curvature and the distance to the ridge are the most important parameters influencing avalanche release area distribution. Failletaz et al. (2006); Fyffe and Zaiser (2004) and Fyffe and Zaiser (2007) used cellular-automata approaches to compute avalanche release area distributions. These models include a source of stochastic variability such as the heterogeneity of the weak layer mechanical properties. Interestingly, these models are able, under certain conditions, to reproduce the power-law area distributions observed from field measurements (McClung, 2003; Failletaz et al., 2004). Finally, Simenhois and Birkeland (2014) recently suggested different mechanisms that may control fracture arrest propensity.

In this paper, we extend a mechanically based probabilistic model developed in a previous study (Gaume et al., 2012, 2013) to analyze which snowpack parameters are influencing slab tensile failure propensity and, hence, the extent of the release area. In the first section, we recall the main characteristics of the model and present the changes made compared to its previous version. Then, in the second section, two release types are distinguished and presented. In the third section, we quantify the influence of the characteristics of weak layer heterogeneity and of slab properties on the position of slab tensile failure. Finally, in the last section, the results are applied to dry-snow slab avalanche release.

\section{Formulation of the model}

In this paper, the mechanical model proposed by Gaume et al. (2012) and described in detail in Gaume et al. (2013) is used and extended. We recall here its main characteristics.

The simulated system is a uniform slope composed of a slab and a weak layer of length $L=50 \mathrm{~m}$. The numerical simulations are carried out using the finite element code Cast $3 \mathrm{~m}$ in 2-D (plane stress condition). Gravity is the only applied external force and the system is loaded by progressively increasing the slope angle $\theta$ until failure. The main change compared to the model of Gaume et al. (2013) concerns the constitutive law of the slab. We use here an elasticbrittle law, characterized by the existence of a tensile strength $\sigma_{\mathrm{t}}$, in order to take into account the possible tensile failure of the slab. The density of the slab is denoted $\rho$. The elasticity of the slab is characterized by its Young's modulus $E$ and its Poisson's ratio v. The weak layer is modeled as a quasi-brittle (strain-softening) interface with a MohrCoulomb failure criterion characterized by a cohesion $c$ and a friction coefficient $\mu$. Spatial heterogeneity of the weak layer is accounted for by a stochastic Gaussian distribution of the cohesion $c$ with a spherical covariance function of correlation length $\epsilon$. The average cohesion is denoted $\langle c\rangle$, its standard deviation $\sigma_{c}$ and the coefficient of variation is calculated by $\mathrm{CV}=\sigma_{c} /\langle c\rangle$. The range of parameter values used in the model, compared to realistic values for snow coming from field and laboratory experiments, is summarized in Table 1.

Besides the evaluation of avalanche release depth distributions, this model formerly enabled us to evidence a heterogeneity smoothing effect caused by stress redistributions due to slab elasticity. This elastic smoothing effect is characterized by a typical length scale of the system $\Lambda$ (see Chiaia et al., 2008; Gaume et al., 2013, 2014a, for more details).

\section{Preliminary description of the results: two release types}

Two types of avalanche release were obtained in the simulations: (1) full-slope release when the entire simulated slope becomes unstable without tensile failure within the slab (Fig. 1a) and (2) partial-slope release when tensile failure occurs within the slab so that only a part of the slope is released (Fig. 1b). Importantly, however, for both release types, the primary failure process observed is always the shear failure of the weak layer. Slab tensile failure, when existent, systematically constitutes a secondary process.

In the case of a full-slope release, the heterogeneity magnitude is not sufficient to trigger a tensile failure within the slab. The basal crack in the weak layer thus propagates until reaching the top boundary condition which can be seen as an anchor point (Fig. 1a). In the context of a real avalanche starting zone, this boundary condition can represent a strong geomorphological feature that may trigger the tensile failure (ridges, rocks, trees, local convex zone, etc.).

In contrast, for partial-slope releases the cohesion variability in the weak layer is sufficient to generate the tensile failure of the slab within the simulated system. Local strong zones can effectively stop the propagation of the crack and the excess of stress is redistributed in the slab and induces slab tensile opening.

For a constant slab failure strength and a constant average cohesion of the WL, the occurrence of full- or partial-slope release is intimately linked to the heterogeneity of WL cohesion as well as smoothing effects due to the elasticity of the slab. These combined mechanisms lead to shear stress heterogeneities in the WL which modulate the shear stress differences, $\Delta \tau$, existing between adjacent elements of the WL. This shear stress difference $\Delta \tau$ ultimately induces generation of normal stresses in the slab in the down-slope direction $\sigma_{x x}$ ( $\sigma_{x x}$ is positive in tension and negative in compression). If $\sigma_{x x}$ exceeds locally the tensile strength of the slab $\sigma_{t}$, then the brittle failure of the slab occurs. This process is illustrated in Fig. 1 for both release types. A full-slope release corresponds 
Table 1. Parameters used in the model and typical values for snow from field and laboratory experiments. For more details about these parameters and a more complete review of the different related studies, see Sect. 3.3.2 in Gaume (2012).

\begin{tabular}{|c|c|c|c|c|}
\hline & Parameter & Modeled values & Typical values & References \\
\hline \multirow{5}{*}{$\frac{\tilde{\pi}}{n}$} & $\rho\left(\mathrm{kg} \mathrm{m}^{-3}\right)$ & $80-250$ & $100-300$ & Schweizer (1999) \\
\hline & $E(\mathrm{MPa})$ & $0.1-20$ & $0.1-50$ & Mellor (1975); Scapozza (2004); Sigrist (2006) \\
\hline & $v$ & 0.2 & $0.1-0.4$ & Ooizumi and Huzioka (1982); Schweizer (1999) \\
\hline & $\sigma_{\mathrm{t}}(\mathrm{kPa})$ & $0-10$ & $0.1-30$ & McClung (1979); Conway and Abrahamson (1984) \\
\hline & & & & Jamieson and Johnston (1990); Sigrist (2006) \\
\hline \multirow{5}{*}{ 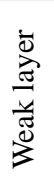 } & $c(\mathrm{kPa})$ & $0.5-1.5$ & $0.1-4$ & Jamieson and Johnston (2001) \\
\hline & $\mathrm{CV}(\mathrm{kPa})$ & $0-0.5$ & $0.2-0.6$ & Föhn (1989); Jamieson and Johnston (2001); Landry (2002) \\
\hline & & & & Kronholm (2004); Logan et al. (2007); Schweizer et al. (2008) \\
\hline & $\epsilon(\mathrm{m})$ & $0-5$ & $<10$ & Schweizer et al. (2008) \\
\hline & $\mu$ & 0.56 & $0.5-0.75$ & van Herwijnen and Heierli (2009) \\
\hline
\end{tabular}

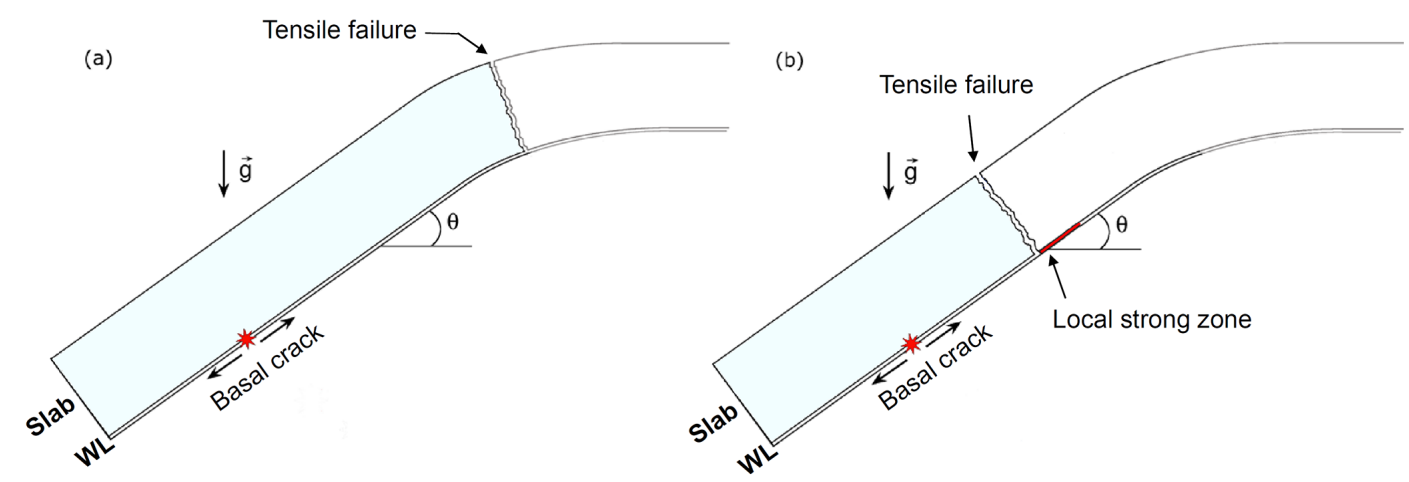

Figure 1. Schematic representing the two types of failure observed in the simulations. (a) Full-slope release: the crack in the WL propagates across the whole slope. The position of the slab tensile failure would then depend on morphological features (rocks, trees, ridges, curvature, etc.). (b) Partial-slope release: the local heterogeneity is sufficient to stop the propagation of the crack in the WL and thus trigger the tensile failure within the slab. The red-colored part of the weak layer represents a local zone of large shear strength.

to $\sigma_{x x}<\sigma_{\mathrm{t}}$ everywhere in the slab, whereas a partial-slope release means that $\sigma_{x x}$ can be locally larger than $\sigma_{\mathrm{t}}$.

Note that the position of the tensile failure in the slab, if existent, also depends on the position of the initial basal failure in the weak layer. This is due to the fact that the stress concentration at the crack tip increases naturally with the crack size. Hence, the shear stress difference $\Delta \tau$ and tensile stresses in the slab $\sigma_{x x}$ depend not only on WL heterogeneity but also on the size of the basal crack.

\section{Quantitative results}

For each set of the model parameters, 100 finite element (FE) simulations were performed for different realizations of the WL heterogeneity with a constant average cohesion $\langle c\rangle$. As explained, each simulation led to either a full-slope or a partial-slope release. Note that due to the WL heterogeneity, the release occurs for different values of the slope angle $\theta$ (see Gaume et al., 2013, for more details). Besides, we only considered cases in which the load was sufficient to trigger the WL failure. Hence, the slab thickness $D$ was chosen higher than the critical thickness $D_{c}=\langle c\rangle /(\rho g)$ to ensure this assumption. The results are presented in terms of partialslope release probability, also called tensile failure probability, $P_{\mathrm{t}}$. This probability represents the probability that $\sigma_{x x}$ exceeds locally (i.e., at the crack tip) the tensile strength of the slab $\sigma_{\mathrm{t}}$ and is thus $P_{\mathrm{t}}=P\left(\sigma_{x x}>\sigma_{\mathrm{t}}\right)$. As the variability of the tensile stress $\sigma_{x x}$ is due to the WL Gaussian heterogeneity, $\sigma_{x x}$ also follows a Gaussian distribution of average $\left\langle\sigma_{x x}\right\rangle$ and standard deviation $\sigma_{\sigma_{x x}}$. Hence, the exceedance probability $P\left(\sigma_{x x}>\sigma_{\mathrm{t}}\right)$ can be analytically expressed and is given by

$$
\begin{aligned}
& P_{\mathrm{t}}=P\left(\sigma_{x x}>\sigma_{\mathrm{t}}\right)=\int_{\sigma_{\mathrm{t}}}^{\infty} p\left(\sigma_{x x}\right) d \sigma_{x x} \\
& =1-\frac{1}{2}\left[1+\operatorname{erf}\left(\frac{\sigma_{\mathrm{t}}-\left\langle\sigma_{x x}\right\rangle}{\sqrt{2} \sigma_{\sigma_{x x}}}\right)\right],
\end{aligned}
$$

where $p\left(\sigma_{x x}\right)$ is the probability density function of $\sigma_{x x}$

In the first part of this section (parametric analysis), the influence of the characteristics of WL heterogeneity (cohesion 


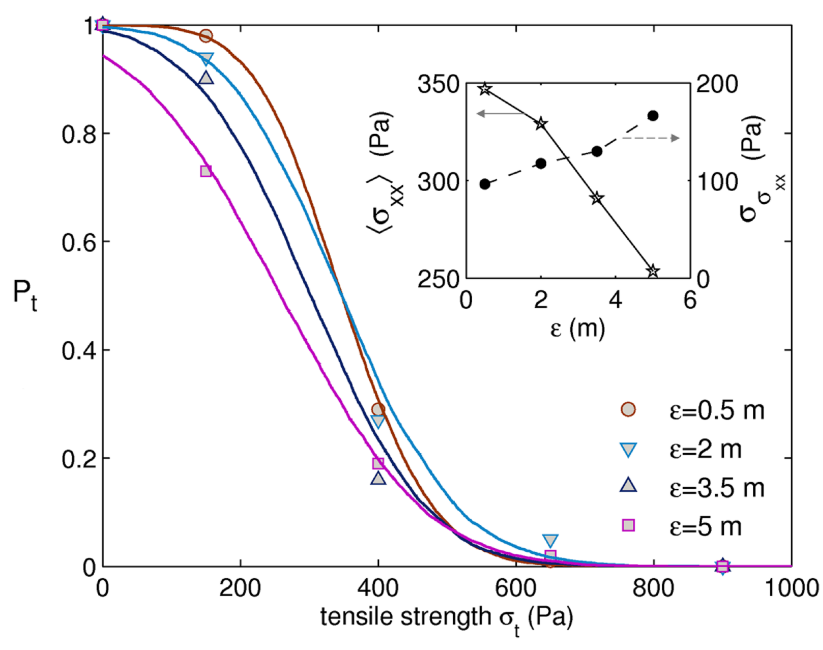

Figure 2. Probability of slab tensile failure $P_{\mathrm{t}}$ within the simulated system (partial-slope release) as a function of the tensile strength $\sigma_{\mathrm{t}}$ for different values of the correlation length $\epsilon$, a coefficient of variation $\mathrm{CV}=0.3$, a constant slab thickness $D=1 \mathrm{~m}$, slab density $\rho=250 \mathrm{~kg} \mathrm{~m}^{-3}$ and Young's modulus $E=1 \mathrm{MPa}$. In the inset, the average tensile stress $\left\langle\sigma_{x x}\right\rangle$ (solid line) and its standard deviation $\sigma_{\sigma_{x x}}$ (dashed line) are represented as a function of $\epsilon$.

standard deviation $\sigma_{\mathrm{c}}$ and correlation length $\epsilon$ ) and the slab properties (slab thickness $D$, tensile strength $\sigma_{\mathrm{t}}$ and Young's modulus $E)$ on $P_{\mathrm{t}}=P\left(\sigma_{x x}>\sigma_{\mathrm{t}}\right),\left\langle\sigma_{x x}\right\rangle$ and $\sigma_{\sigma_{x x}}$ is shown. The influence of each parameter is studied while the other parameters are kept constant. Then, in the second part of this section (application to slab avalanche release), the relations existing between the different mechanical properties of snow are taken into account.

\subsection{Parametric analysis}

\subsubsection{Tensile strength $\sigma_{\mathrm{t}}$}

Figures 2, 3a and 4a represent the probability of tensile failure $P_{\mathrm{t}}$ within the system as a function of the tensile strength $\sigma_{\mathrm{t}}$ for different values of the correlation length $\epsilon$ (Fig. 2), the coefficient of variation CV (Fig. 3a) and the slab thickness $D$ (Fig. 4a). Tensile strength varies between 0 and $1 \mathrm{kPa}$. As expected, this probability systematically decreases from 1 to 0 with increasing tensile strength $\sigma_{\mathrm{t}}$. The data points obtained with the FE simulations have been fitted using Eq. (1), allowing us to compute the average tensile stress $\left\langle\sigma_{x x}\right\rangle$ and its standard deviation $\sigma_{\sigma_{x x}}$ (which are the two parameters of the fit). The good agreement between the modeled exceedance probability $P_{\mathrm{t}}$ (Eq. 1) and the FE simulation results confirm that $\sigma_{x x}$ follows a normal distribution resulting from the Gaussian WL heterogeneity.

\subsubsection{Correlation length $\epsilon$ of WL cohesion}

The influence of the correlation length $\epsilon$ is shown in Fig. 2 . The tensile failure probability $P_{\mathrm{t}}$ decreases in general with increasing correlation length $\epsilon$. The average tensile stress $\left\langle\sigma_{x x}\right\rangle$ decreases with $\epsilon$ while its standard deviation $\sigma_{\sigma_{x x}}$ increases. The influence of $\epsilon$ is thus more pronounced for small values of the tensile strength $\sigma_{\mathrm{t}}$. Hence the higher the correlation length, the farther a crack propagates, leading to more full-slope releases and thus potentially larger avalanches.

The correlation length $\epsilon$ characterizes the spatial structure of the cohesion heterogeneity. A nil correlation length corresponds to a completely random signal, whereas a large correlation length indicates the existence of spatial structures in the signal with similar values (see Gaume et al., 2013, for some examples). Hence, an increase of the correlation length smooths the WL heterogeneity and reduces the shear stress difference $\Delta \tau$ between two elements, resulting in a lower fracture arrest propensity. In contrast, a very low value of the correlation length implies a WL heterogeneity without spatial structure and thus large local variations that can stop the propagation of the crack. This observation qualitatively explains the observed trend of the results.

\subsubsection{Standard deviation $\sigma_{\mathrm{c}}$ of WL cohesion}

The influence of the standard deviation $\sigma_{\mathrm{c}}$ is then investigated and represented in Fig. 3. For a constant slab thickness $D=1 \mathrm{~m}$ and correlation length $\epsilon=0.5 \mathrm{~m}$, Fig. 3a shows, as would be expected, the increase of the tensile failure probability with increasing coefficient of variation $\mathrm{CV}=\sigma_{\mathrm{c}} /\langle c\rangle$. This dependence is even clearer in Fig. $3 \mathrm{~b}$ in which $P_{\mathrm{t}}$ is represented as a function of $\mathrm{CV}$ for $\sigma_{t}=100 \mathrm{~Pa}$ and different slab thickness values. Inset of Fig. 3a also shows that the average tensile stress $\left\langle\sigma_{x x}\right\rangle$ as well as its standard deviation $\sigma_{\sigma_{x x}}$ linearly increase with the coefficient of variation, explaining why the curves in Fig. 3a are progressively shifted to the right with a lower rate of decrease with $\sigma_{\mathrm{t}}$ as the coefficient of variation increases.

Indeed, a large value of the coefficient of variation induces large local variations of the WL shear stress, resulting in high tensile stresses within the slab, and ultimately favors fracture arrest. As a consequence, the tensile failure probability increases with increasing variability.

\subsubsection{Slab thickness $D$}

As shown in Fig. $4 \mathrm{a}$, the tensile failure probability $P_{\mathrm{t}}$ in general decreases with increasing slab thickness $D$. Furthermore, the higher $D$ is, the faster the probability decreases with $\sigma_{\mathrm{t}}$. The values of $\sigma_{\mathrm{t}}$ for $P_{\mathrm{t}}=1$ are almost unaffected by the slab thickness $D$ while the value for $P_{\mathrm{t}}=0$ decreases with increasing $D$. These characteristics correspond to the relations of $\left\langle\sigma_{x x}\right\rangle$ and $\sigma_{\sigma_{x x}}$ with the slab thickness $D$, which both decrease with increasing thickness. Note that, as men- 



Figure 3. (a) Probability of slab tensile failure $P_{\mathrm{t}}$ within the simulated system (partial-slope release) as a function of the tensile strength $\sigma_{\mathrm{t}}$ for different values of the coefficient of variation $\mathrm{CV}$ and for a constant correlation length $\epsilon=2 \mathrm{~m}$, a constant slab thickness $D=1 \mathrm{~m}$, slab density $\rho=250 \mathrm{~kg} \mathrm{~m}^{-3}$ and a Young modulus $E=1 \mathrm{MPa}$. In the inset, the average tensile stress $\left\langle\sigma_{x x}\right\rangle$ and its standard deviation $\sigma_{\sigma_{x x}}$ are represented as a function of CV. (b) $P_{\mathrm{t}}$ is represented as a function of $\mathrm{CV}$ for different slab thickness values, a tensile strength $\sigma_{\mathrm{t}}=100 \mathrm{~Pa}$ and the same value of the other parameters as in (a). In the inset, the tensile failure probability $P_{\mathrm{t}}$ is represented vs. the standard deviation scaled by $f_{\mathrm{c}}(D)=0.64 D-0.17$ (in $\mathrm{m}$ ).

tioned before, simulations were carried out for $D>D_{\mathrm{c}}=$ $c /(\rho g)=0.41 \mathrm{~m}$ in this case to ensure that a WL shear failure can be initiated. Indeed, if no failure is initiated in the $\mathrm{WL}$, no slab tensile failure can occur.

In more detail, Fig. $4 \mathrm{~b}$ reports the tensile failure probability $P_{\mathrm{t}}$ directly as a function of $D$ for different tensile strength values. For $\sigma_{\mathrm{t}}<150 \mathrm{~Pa}, P_{\mathrm{t}}$ is approximately equal to 1 , whereas it is approximately equal to 0 for $\sigma_{\mathrm{t}}>900 \mathrm{~Pa}$. For intermediate values of $\sigma_{\mathrm{t}}$, the tensile failure probability generally decreases from $D=0.5 \mathrm{~m}$ to $D=2 \mathrm{~m}$.
It has previously been shown (Gaume et al., 2013, 2014a) that the slab thickness $D$, together with the slab elastic modulus $E$, has an important smoothing effect on WL spatial variability due to stress redistribution. Consequently, a large value of the slab thickness $D$ smooths the cohesion heterogeneity by reducing the apparent standard deviation of the WL heterogeneity, in contrast to the case of a completely rigid slab in which the stresses would follow exactly the heterogeneity variations. As shown before, a reduction of the standard deviation leads to a decrease of the tensile failure probability, which explains the results described above. This result is also clearly illustrated in Fig. 3b. The rate of increase of the tensile failure probability $P_{\mathrm{t}}$ decreases with the slab thickness. Hence, as the slab thickness increases, the WL heterogeneity is smoothed and thus a larger value of $\mathrm{CV}$ is required to obtain $P_{t}=1$, i.e., only partial-slope releases. As shown in the inset, the smoothing effect by the slab thickness can be accounted for simply through a scaling of the standard deviation with a linear function of the slab thickness $\rho g f_{\mathrm{c}}(D)$ with $f_{\mathrm{c}}(D)=0.64 D-0.17$ (in m).

\subsubsection{Young's modulus $E$ of slab}

Figure 5 represents the influence of the slab Young's modulus on the tensile failure probability $P_{\mathrm{t}}$ for a correlation length $\epsilon=2 \mathrm{~m}$, a slab thickness $D=1 \mathrm{~m}$ and a slab tensile strength $\sigma_{\mathrm{t}}=600 \mathrm{~Pa}$. The tensile failure probability increases with the slab Young's modulus $E$. Indeed, because the slab is brittle elastic, the tensile stress $\sigma_{x x}$ increases linearly with the deformation in the down-slope direction $\epsilon_{x x}$ according to $\sigma_{x x}=E^{\prime} \epsilon_{x x}$ until $\sigma_{x x}$ reaches the tensile strength $\sigma_{\mathrm{t}}$ (with $E^{\prime}=E /\left(1-v^{2}\right)$ ). Hence for a constant deformation $\epsilon_{x x}$ which is imposed by the displacement gradient in the WL interface and thus by the WL stress variations, the tensile stress is lower in the case of a low Young's modulus than for a high one (see insets in Fig. 5). As a consequence, the slab failure criterion, namely $\sigma_{x x}=\sigma_{\mathrm{t}}$, is met easily in the limiting case of a rigid slab.

Moreover, as shown in Gaume et al. (2013, 2014a), slab elasticity also induces an important smoothing effect on WL heterogeneity. This effect leads to a reduction of the apparent standard deviation which, together with the previous explanation, explains the decrease of the tensile failure propensity with increasing elasticity (decreasing Young's modulus $E$ ).

\subsection{Application to slab avalanche release}

The results of the previous parametric analysis should be interpreted with care and one should keep in mind that, for snow, several of the previous parameters are linked which may lead to more complex interactions. For instance, the result of the influence of Young's modulus on the tensile failure probability might seem contradictory to avalanche observations. Indeed, taken as it is, this result would imply that it is easier to trigger a tensile failure in stiff and strong snow than 

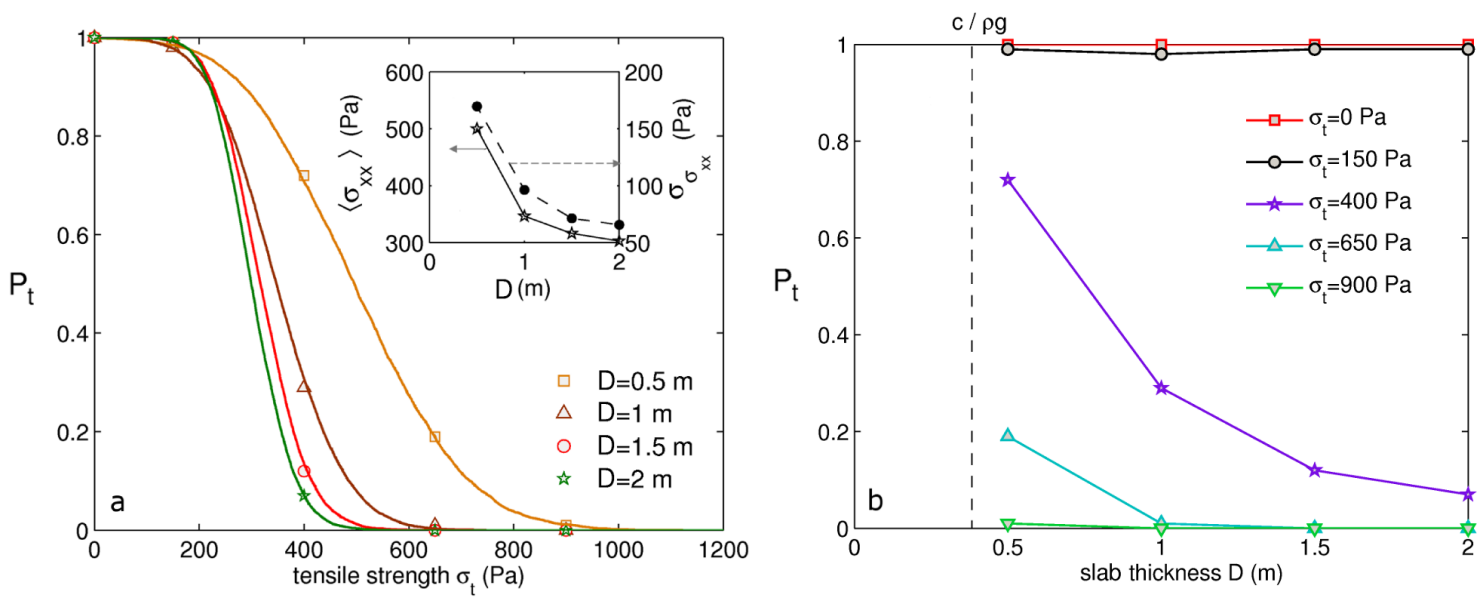

Figure 4. (a) Probability of slab tensile failure $P_{\mathrm{t}}$ within the simulated system (partial-slope release) as a function of the tensile strength $\sigma_{\mathrm{t}}$ for different slab thickness values and a constant correlation length $\epsilon=0.5 \mathrm{~m}$, slab density $\rho=250 \mathrm{~kg} \mathrm{~m}^{-3}$ and Young's modulus $E=1 \mathrm{MPa}$. In the inset, the average tensile stress $\left\langle\sigma_{x x}\right\rangle$ and its standard deviation $\sigma_{\sigma_{x x}}$ are represented as a function of $D$. (b) $P_{\mathrm{t}}$ is represented as a function of $D$ for different tensile strength values and the same value of the other parameters as in (a).

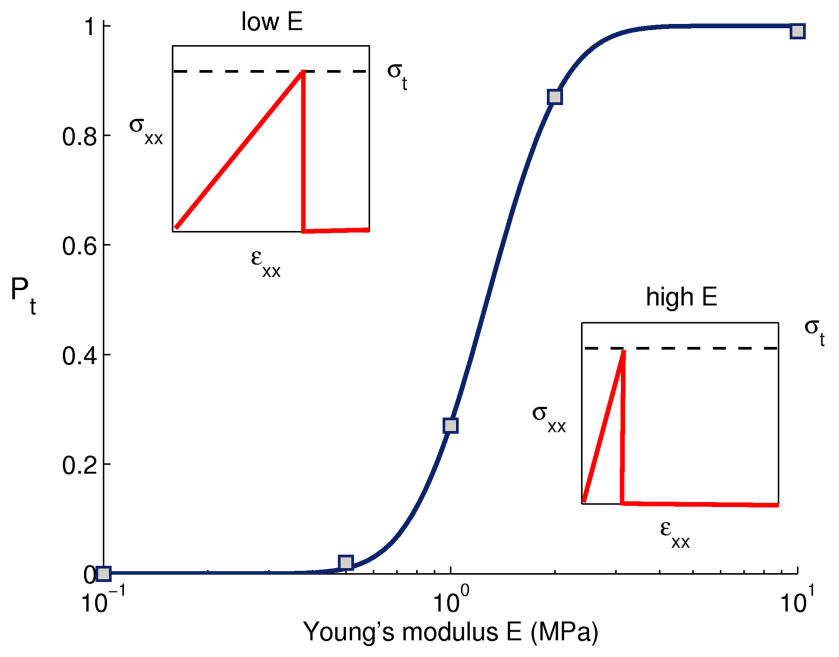

Figure 5. Slab tensile failure probability $P_{\mathrm{t}}$ vs. Young's modulus of the slab for a correlation length $\epsilon=2 \mathrm{~m}$, a slab thickness $D=1 \mathrm{~m}$, a slab density $\rho=250 \mathrm{~kg} \mathrm{~m}^{-3}$ and a tensile strength $\sigma_{\mathrm{t}}=400 \mathrm{~Pa}$. The insets show illustrations of the tensile stress $\sigma_{x x}$ vs. down-slope deformation $\epsilon_{x x}$ for a low (left) and a high Young's modulus (right).

in softer snow. If this line of reasoning is pursued, hard snow slabs would result in smaller avalanche size than soft slabs, which is clearly in contradiction to avalanche observations. Hence, even if the result behind Fig. 5 is consistent, from a mechanical point of view it cannot be directly applied to dry-snow slab avalanche release. To do so, one needs to take into account the relation between the density of the slab $\rho$, its Young's modulus $E$ and its tensile strength $\sigma_{\mathrm{t}}$.
New simulations were therefore performed, for which the dependence among $\rho, E$ and $\sigma_{\mathrm{t}}$ was taken into account using the relation proposed by Sigrist (2006):

$E(\rho)=9.68 \times 10^{8}\left(\frac{\rho}{\rho_{\text {ice }}}\right)^{2.94}$

and

$\sigma_{\mathrm{t}}(\rho)=2.4 \times 10^{5}\left(\frac{\rho}{\rho_{\text {ice }}}\right)^{2.44}$,

where $\rho_{\text {ice }}=917 \mathrm{~kg} \mathrm{~m}^{-3}$. The density of the slab was varied between 80 and $250 \mathrm{~kg} \mathrm{~m}^{-3}$, leading to values of Young's modulus between 0.7 and $20 \mathrm{MPa}$ and tensile strength between 0.5 and $10 \mathrm{kPa}$. Two types of simulations were performed: (1) simulations with a constant slab thickness and therefore a different slab mass for the different densities and (2) simulations with a slab thickness which was varied in order to keep a constant load on the WL for the different densities.

In both cases, the slab tensile failure probability $P_{\mathrm{t}}$ decreases as a function of slab density when considering the above mentioned interdependencies (Fig. 6). Besides, it appears that for densities larger than about $150 \mathrm{~kg} \mathrm{~m}^{-3}$, the tensile failure probability becomes very small, meaning that all the releases are full slope. Thus, for large enough densities, the WL layer heterogeneity has no influence on the position and the extent of the avalanche release area. In this case, this result would suggest that topographical and geomorphological features control the size of the release area. This result is illustrated below the graphic by two cases of avalanches: (left) a soft and shallow slab for which the release area is quite small compared to the maximum potential extent of this 

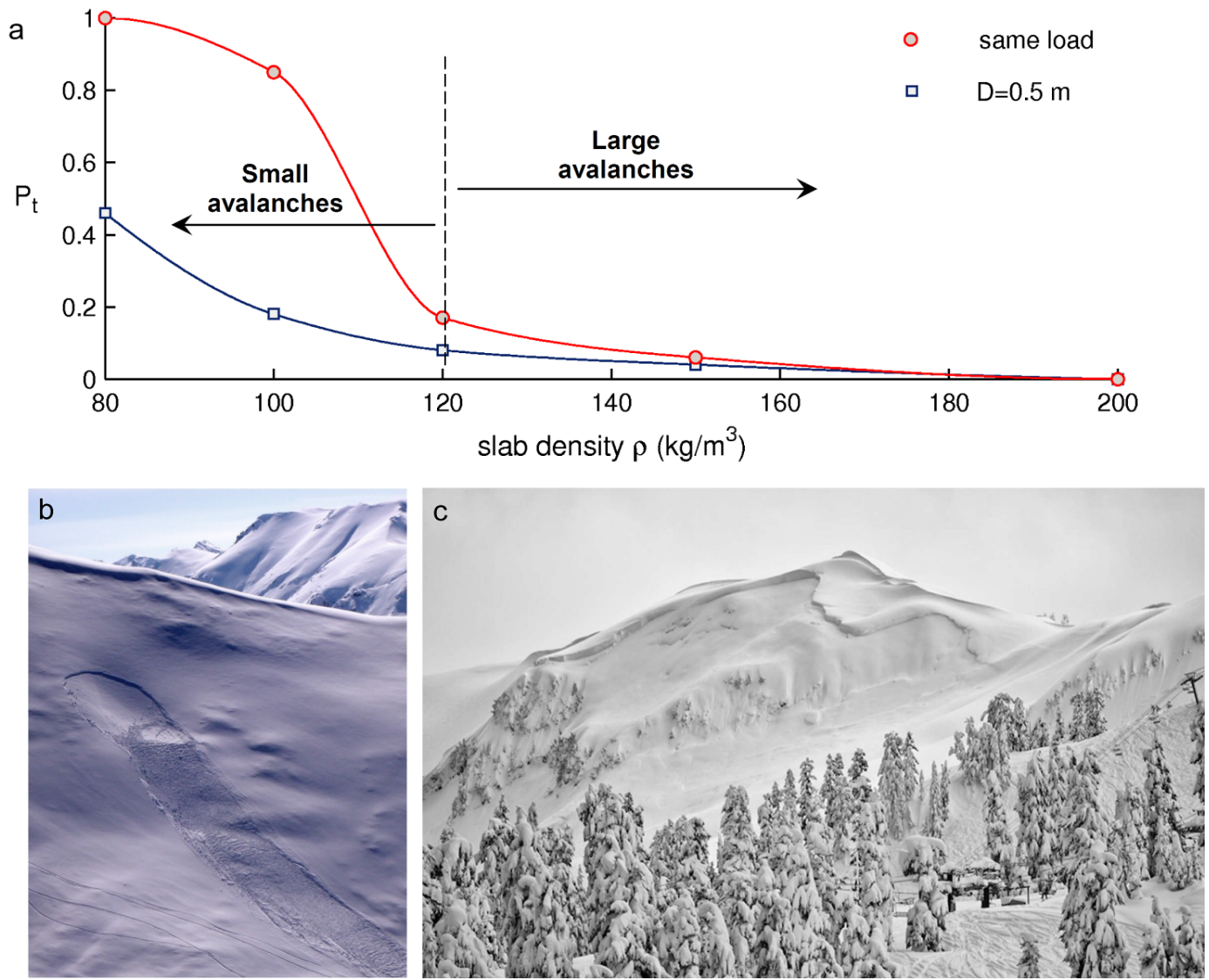

Figure 6. Slab tensile failure probability $P_{\mathrm{t}}$ vs. slab density for $\epsilon=2 \mathrm{~m}$ and taking into account the relation between slab density $\rho$, its Young's modulus $E$ and its tensile strength $\sigma_{\mathrm{t}}$ according to Eqs. (2) and (3). The red curve represents the results of simulations for a constant load and thus a slab thickness which decreases with slab density $\left(\rho D=100 \mathrm{~kg} \mathrm{~m}^{-2}\right)$. The blue curve represents a constant slab thickness $D=0.5 \mathrm{~m}$ and thus a varying load. Below are two avalanche pictures illustrating the result: (left) soft and shallow slab of small size $\left({ }^{\circledR}\right.$ Anchorage Avalanche Center); (right) hard and thick slab with a very large extent ( ${ }^{\odot}$ Grant Gunderson).

starting zone and (right) a hard and thick slab for which the release area is very extensive and controlled by terrain features. In the latter case, the tensile failure generally occurs at the transition between slope angles larger and smaller than $30^{\circ}$ due to the basal friction (the crack face friction angle of snow being around $30^{\circ}$; van Herwijnen and Heierli, 2009).

\section{Discussion}

The proposed approach allows us to compute the slab tensile failure probability from WL spatial variability characteristics and slab properties using the finite element method. First, a parametric analysis showed the influence of each model parameter on the tensile failure probability. Then, more realistic simulations were performed, taking into account the link between the mechanical properties of the slab. These simulations explained why hard and thick snow slabs are more prone to wide-scale crack propagation than soft slabs. However, one might also argue that the density is generally linked to the thickness: the higher the thickness, the higher the den- sity due to settlement. Nevertheless, even if this link was taken into account, the main finding of Fig. 6, namely that the WL heterogeneity influences fracture arrest propensity only for soft slabs, would still remain relevant since the tensile failure probability would be even lower. Indeed, as shown in Fig. 4, an increase in slab thickness decreases the tensile failure probability, and thick slabs thus lead to larger release areas by smoothing the WL heterogeneity. Furthermore, since the WL was modeled as an interface, the bending of the slab observed in field propagation saw tests (van Herwijnen et al., 2010), which can increase the tensile stress (Gaume et al., 2014b; Schweizer et al., 2014), was not taken into account. This bending effect would probably induce a higher transition density since more partial-slope releases would occur.

From the presented approach, a rough estimate of the avalanche release area can also be proposed. For a tensile failure probability equal to zero, the avalanche release area would be equal to the maximum area $A_{\max }$ allowed by the terrain or the snow cover distribution. Contrarily, for a tensile failure probability equal to 1 , the avalanche release area 


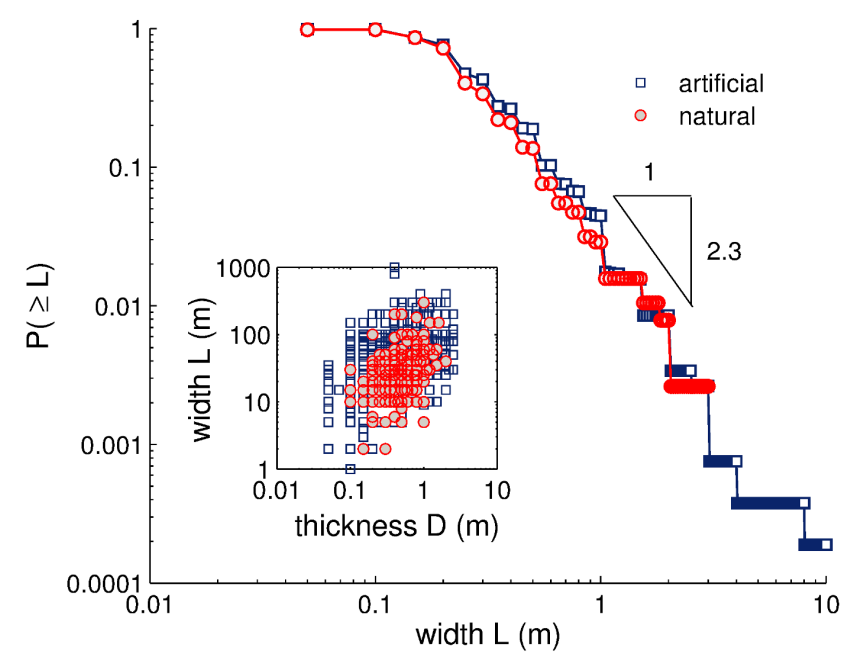

Figure 7. Cumulative exceedance probability of the width $L$ of 369 natural and 5323 artificially triggered slab avalanches observed during winters 1998 to 2010 in La Plagne (France). The inset shows the width $L$ vs. the release thickness $D$.

would be close to zero. Hence, the release area $A$ can be approximated in a first approach by

$A=\left(1-P\left(\sigma_{\mathrm{xx}}>\sigma_{\mathrm{t}}\right)\right) A_{\max }$.

The avalanche release area would thus be different from $A_{\max }$ only for very soft slabs, in which case it would also be a function of the characteristics of WL heterogeneity and slab properties. The form of the proposed estimate of the release area is in good quantitative agreement with the result of Fyffe and Zaiser (2007), who also studied the influence of WL heterogeneity parameters on the size of the release zone using a cellular automaton model. These authors showed that the release area increases with the tensile strength of the slab and then tends to a limit given by the maximum size of the system for a tensile strength $\sigma_{\mathrm{t}} \approx 4000 \mathrm{~Pa}$ (calculated using the dimensionless analysis proposed by Fyffe and Zaiser, 2007, and the parameters of our model) corresponding to a density $\rho \approx 170 \mathrm{~kg} \mathrm{~m}^{-3}$.

Figure 7 reports the exceedance probability of the width $L$ of 369 natural and 5323 artificially triggered slab avalanches that were observed during the winters 1998 to 2010 in La Plagne (France). These data were presented in detail in Gaume et al. (2012), in which the focus was on the avalanche release thickness rather than the width. The inset shows that the avalanche width $L$ is not very well correlated with the release thickness $D$ despite a relatively slight apparent increasing trend which is statistically not significant $\left(R^{2}=0.15\right.$ for a linear regression). Our numerical results suggest that $L$ should not be correlated with $D$ at all except for very low densities and thus small avalanches. However, as mentioned before, bending effects induced by WL failure (which were not accounted for) would tend to increase the tensile failure probability and consequently the dependency of $L$ with
$D$. Furthermore, the roughness of the terrain is progressively smoothed during the season as the slab thickness increases. Hence, the avalanche maximum extent $A_{\max }$ also potentially increases with increasing slab thickness $D$ (Veitinger et al., 2014). Besides, we remark that the exceedance probability $P(\geq L)$ does not appear to depend on the avalanche triggering mode, natural or artificial. Hence the width of the release area is essentially uninfluenced by the triggering mode. Contrarily, Gaume et al. (2012) showed an important difference between natural and artificial triggering on the release depth distribution: natural avalanches are influenced not only by terrain and mechanical aspects but also recent snowfall distributions. This similarity between the width distributions of naturally and artificially triggered avalanches confirms that the release area is mainly influenced by terrain characteristics (possibly smoothed by the snow cover distribution; Veitinger et al., 2014) and slab properties (density and thickness).

Finally, the results of the presented model suggest that the majority of the releases would be full slope, i.e., not influenced by WL heterogeneity, especially for high densities. Hence, the potential extent of slab avalanche release areas will be controlled by topographical and geomorphological features of the path such as rocks, trees, ridges or local curvatures induced by the terrain and the snow cover distribution. As a consequence, GIS methods based on terrain characteristics such as those developed by Maggioni and Gruber (2003) might be adequate to compute the potential extent of extreme avalanches. For more frequent avalanches, similar and recent methods also taking into account the spatial distribution of the snow depth and the induced terrain smoothing would be relevant (Veitinger et al., 2014).

\section{Conclusions}

We used a coupled mechanical-statistical approach to study the probability of the occurrence of slab tensile failure of a slab-WL system using the finite element method. Two different release types were observed in the simulations: (1) Fullslope release when the WL heterogeneity is not sufficient to arrest crack propagation and trigger a tensile failure within the slab, and hence the crack propagates across the whole system; (2) partial-slope release when the local variations of WL cohesion are substantial and can stop crack propagation and trigger the slab tensile failure. Importantly, for both release types the primary failure process observed is always the basal shear failure of the weak layer. Hence slab fracture systematically constitutes a secondary process.

We have shown that the slab tensile failure propensity strongly depends on the model parameters such as the tensile strength $\sigma_{\mathrm{t}}$, the slab thickness $D$, the correlation length $\epsilon$, the standard deviation of the weak layer cohesion $\sigma_{\mathrm{c}}$ and probably other parameters that have not been varied in this study such as the average cohesion $\langle c\rangle$. In addition, we presented a simple statistical model capable of reproducing the 
tensile failure probability as a function of the model parameters. Two illustrations of this simple model are represented in Fig. 1. In the first case (Fig. 1a), the slab tensile stress $\sigma_{x x}$ is always lower than the tensile strength $\sigma_{\mathrm{t}}$. The basal failure thus propagates across the entire system up to the top boundary condition, which can be seen as a ridge, a rock, a tree or a local curvature. In the second case (Fig. 1b), a local zone of substantial tensile stress $\sigma_{x x}$ due to strong variation of the WL cohesion generates a local tensile failure within the slab since $\sigma_{x x}>\sigma_{\mathrm{t}}$.

For realistic values of the parameters and taking the link between the mechanical properties of the slab into account, the model results suggest that the releases are partial slope only for low slab densities and full slope for densities higher than about $150 \mathrm{~kg} \mathrm{~m}^{-3}$. Hence, in most cases one would expect that the extent of the release area is mainly controlled by the topography and the morphology of the path. This finding corroborates the results found by Maggioni and Gruber (2003), who analyzed the influence of morphological features of the path on the extent of the release area using a purely data-driven statistical approach, and provides some mechanical justification to the predominance of local terrain geometry in the position and extent of real avalanches. This result shows in particular that the release area will be extremely dependent on slope topography (local curvature, ridge, etc.), on the presence of rocks and trees, for instance, and also on the snow cover distribution which can induce terrain smoothing and thus promote wide-scale crack propagation.

Acknowledgements. We wish to express our gratitude to Claude Schneider, snow expert in La Plagne, for providing the database of avalanche sizes. J. Gaume was supported by a Swiss Excellence Government Scholarship and is grateful for support by the State Secretariat for Education, Research and Innovation (SERI) of the Swiss government. We thank two anonymous reviewers for their constructive comments that helped to improve our paper.

Edited by: F. Dominé

\section{References}

Ancey, C., Gervasoni, C., and Meunier, M.: Computing extreme avalanches, Cold Reg. Sci. Technol., 39, 161-180, 2004.

Barbolini, M., Gruber, U., Keylock, C., Naaim, M., and Savi, F.: Application and evaluation of statistical and hydraulic-continuum dense-snow avalanche models to five real European sites, Cold Reg. Sci. Technol., 31, 133-149, 2000.

Chiaia, B., Cornetti, P., and Frigo, B.: Triggering of dry snow slab avalanches: stress vs. fracture mechanical approach, Cold Reg. Sci. Technol., 53, 170-178, 2008.

Conway, H. and Abrahamson, J.: Snow stability index, J. Glaciol., 30, 321-327, 1984.
Eckert, N., Parent, E., and Richard, D.: Revisiting statisticaltopographical methods for avalanche predetermination: Bayesian modelling for runout distance predictive distribution, Cold Reg. Sci. Technol., 49, 88-107, 2007.

Eckert, N., Parent, E., Naaim, M., and Richard, D.: Bayesian stochastic modelling for avalanche predetermination: from a general system framework to return period computations, SERRA, 22, 185-206, 2008.

Eckert, N., Naaim, M., and Parent, E.: Long-term avalanche hazard assessment with a Bayesian depth-averaged propagation model, J. Glaciol., 56, 563-586, 2010.

Failletaz, J., Louchet, F., and Grasso, J.: Two-threshold model for scaling laws of noninteracting snow avalanches, Phys. Rev. Lett., 93, 208001, 2004.

Failletaz, J., Louchet, F., and Grasso, J.: Cellular automaton modelling of slab avalanche triggering mechanisms: from the universal statistical behaviour to particular cases, Proceedings of the ISSW, 174-180, 2006.

Föhn, P.: Snow cover stability tests and the areal variability of snow strength, ISSW Proc., Whistler, BC, Canada, 262-273, 1989.

Föhn, P., Camponovo, C., and Krüst, G.: Mechanical and structural properties of weak snow layers measured in situ, Ann. Glaciol., 26, 1-6, 1998.

Fyffe, B. and Zaiser, M.: The effects of snow variability on slab avalanche release, Cold Reg. Sci. Technol., 40, 229-242, 2004.

Fyffe, B. and Zaiser, M.: Interplay of basal shear fracture and slab rupture in slab avalanche release, Cold Reg. Sci. Technol., 49, 26-38, 2007.

Gaume, J.: Evaluation of avalanche release depths. Combined statistical - mechanical modeling, Ph.D. thesis, Grenoble University, 2012.

Gaume, J., Chambon, G., Eckert, N., and Naaim, M.: Relative influence of mechanical and meteorological factors on avalanche release depth distributions., Geophys. Res. Lett., 39, L12401, doi:10.1029/2012GL051917, 2012.

Gaume, J., Chambon, G., Eckert, N., and Naaim, M.: Influence of weak-layer heterogeneity on snow slab avalanche release: Application to the evaluation of avalanche release depths., J. Glaciol., 59, 423-437, 2013

Gaume, J., Schweizer, J., van Herwijnen, A., Chambon, G., Reuter, B., Eckert, N., and Naaim, M.: Evaluation of slope stability with respect to snowpack spatial variability, J. Geophys. Res., 119, 1783-1789, doi:10.1002/2014JF003193, 2014a.

Gaume, J., van Herwijnen, A., Schweizer, J., Chambon, G., and Birkeland, K.: Discrete element modeling of crack propagation in weak snowpack layers, edited by: Haegeli, P., 2014 International Snow Science Workshop, Banff, Alberta, 2014b.

Jamieson, B. and Johnston, C.: In-situ tensile tests of snowpack layers, J. Glaciol., 36, 102-106, 1990.

Jamieson, B. and Johnston, C.: Refinements to the stability index for skier-triggered dry-slab avalanches, Ann. Glaciol., 26, 296-302, 1998.

Jamieson, J. and Johnston, C.: Evaluation of the shear frame test for weak snowpack layers, Ann. Glaciol., 32, 59-69, 2001.

Kronholm, K.: Spatial variability of snow mechanical properties with regards to avalanche formation, Ph.D. thesis, University of Zurich, 2004. 
Landry, C.: Spatial variations in snow stability on uniform slopes: Implications for extrapolation to surrounding terrain, Master's thesis, Montana State University, USA, 2002.

Logan, S., Birkeland, K., Kronholm, K., and Hansen, K.: Temporal changes in the slope-scale spatial variability of the shear strength of buried surface hoar layers, Cold Reg. Sci. Technol., 47, 148158, 2007.

Maggioni, M. and Gruber, U.: The influence of topographic parameters on avalanche release dimension and frequency, Cold Reg. Sci. Technol., 37, 407-419, 2003.

Maggioni, M., Gruber, U., and Stoffel, M.: Definition and characterisation of potential avalanche release areas, Proceedings of the ESRI Conference, San Diego, 2002.

McClung, D.: Shear fracture precipitated by strain softening as a mechanism of dry slab avalanche release, J. Geophys. Res., 84, 3519-3526, 1979.

McClung, D.: Size scaling for dry snow slab release, J. Geophys. Res., 108, 2465-2477, 2003.

Mellor, M.: A review of basic snow mechanics, IAHS Publication, 114, 251-291, 1975.

Naaim, M., Faug, T., and Naaim-Bouvet, F.: Dry granular flow modelling including erosion and deposition, Surveys in Geophysics, 24, 569-585, 2003.

Ooizumi, M. and Huzioka, T.: Studies of the behavior of a snow cover on a mountain slope, II. Poisson's ratio of snow, Low Temp. Sci. Ser. A, 41, 43-53, 1982.

Salm, B., Burkard, A., and Gubler, H.: Berechnung von Fliesslawinen: eine Anleitung für Praktiker, Swiss Federal Institute for Snow and Avalanche Research SLF, Davos, Switzerland, Mitteilung 47, 37 pp., (in German), 1990.

Scapozza, C.: Entwicklung eines dichte- und temperaturabhängigen Stoffgesetzes zur Beschreibung des visko-elastischen Verhaltens von Schnee, Ph.D. thesis, ETH Zürich, 2004.

Schweizer, J.: Review of dry snow slab avalanche release, Cold Reg. Sci. Technol., 30, 43-57, 1999.

Schweizer, J., Jamieson, B., and Schneebeli, M.: Snow avalanche formation, Rev. Geophys., 41, 1016, doi:10.1029/2002RG000123, 2003.
Schweizer, J., Bellaire, S., Fierz, C., Lehning, M., and Pielmeier, C.: Evaluating and improving the stability predictions of the snow cover model SNOWPACK, Cold Reg. Sci. Technol., 46, 52-59, 2006.

Schweizer, J., Kronholm, K., Jamieson, J., and Birkeland, K.: Review of spatial variability of snowpack properties and its importance for avalanche formation, Cold Reg. Sci. Technol., 51, 253272, 2008.

Schweizer, J., Reuter, B., van Herwijnen, A., Jamieson, B., and Gauthier, D.: On how the tensile strength of the slab affects crack propagation propensity, in: P. Haegeli (Editor), Proceedings ISSW 2014, International Snow Science Workshop, Banff, Alberta, Canada, 29 September-3 October 2014, 164-168, 2014.

Sigrist, C.: Measurements of fracture mechanical properties of snow and application to dry snow slab avalanche release, Ph.D. thesis, ETH Zürich, 2006.

Sigrist, C. and Schweizer, J.: Critical energy release rates of weak snowpack layers determined in field experiments, Geophys. Res. Lett., 34, L03502, doi:10.1029/2006GL028576, 2007.

Simenhois, R. and Birkeland, K. W.: Observations of fracture arrest at slab avalanche boundaries, in: 2014 International Snow Science Workshop, edited by: Haegeli, P., Banff, Alberta, 2014.

van Herwijnen, A. and Heierli, J.: Measurements of crack-face friction in collapsed weak snow layers, Geophys. Res. Lett., 36, L23502, doi:10.1029/2009GL040389, 2009.

van Herwijnen, A., Schweizer, J., and Heierli, J.: Measurement of the deformation field associated with fracture propagation in weak snowpack layers, J. Geophys. Res., 115, doi:10.1029/2009JF001515, 2010.

Veitinger, J., Sovilla, B., and Purves, R. S.: Influence of snow depth distribution on surface roughness in alpine terrain: a multi-scale approach, The Cryosphere, 8, 547-569, doi:10.5194/tc-8-5472014, 2014. 